\title{
Electrodynamic system for two-stage THz-generator on the base of two-channel planar FEM
}

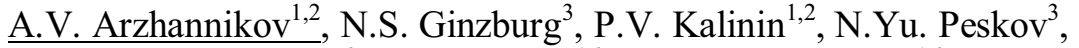 \\ S.E. Sandalov ${ }^{2}$, S.L. Sinitsky ${ }^{1,2}$, and V.D. Stepanov ${ }^{1,2}$ \\ ${ }^{1}$ Budker Institute of Nuclear Physics SB RAS, Novosibirsk, Russia, sinitsky@inp.nsk.su \\ ${ }^{2}$ Novosibirsk State University, Novosibirsk, Russia, \\ ${ }^{3}$ Institute of Applied Physics, RAS, Nizhny Novgorod, Russia
}

\section{Introduction}

Two-dimensional (2-D) distributed feedback gives the opportunity to create generators of a coherent flowof electromagnetic radiation with very wide cross-section. We study such mechanism of spatial synchronization of electromagnetic oscillations for the case of a planar free electron maser (FEM) at the ELMI-device (BINP, Novosibirsk) [1]. To produce high-power THz-radiation few schemes of two-stage generators basing on this mechanism were suggested and investigated $[2,3]$. Theory predictsMW-power levelin THz-radiation pulse [3].To get the first experience the simple two-stage scheme similar to our experiments on mm-wave generation in twochannel planar FEM [4] was chosen. The idea is to use single channel of this planar FEM driven by the first sheet beam as a low frequency (LF) section of $\mathrm{THz}$ generator in which the following parameters of the radiation pulses have been achieved: frequency $\sim 75 \mathrm{GHz}$, spectrum width $\sim 20 \mathrm{MHz}$, pulse duration $\sim 200 \mathrm{~ns}$ and power from single channel $\sim 20 \mathrm{MW}$. This pulse is planned to be delivered to the high frequency (HF) section by means of Bragg wave deflector. After transformation of the pulse to the cutoff wave in the wave transformer it will be scattered by the second sheet e-beam with the frequency conversion to $1 \mathrm{THz}$ band. The parameters and technical solutions for the elements of electrodynamic system obtained on the base of 3D-modeling and tested in "cold" experiments will be presented in the paper.

\section{Electrodynamic System for Two-Stage Generator}

The design of electrodynamic systemincludes two planar channelswith the length of $1.1 \mathrm{~m}$ separated by a metal baffle (see Fig. 1). Two sheet e-beams $(1 \mathrm{MeV} / 1 \mathrm{kA} / 5 \mu \mathrm{s})$ are transported in a guiding uniform magnetic field $B \leq 1.7 \mathrm{~T}$ inside the lower (crosssection $0.95 \times 9 \mathrm{~cm})$ and the upper $(0.6 \times 9 \mathrm{~cm})$ vacuum channels referred as LF- and HF- sections respectively. Besides that, the LF-section is placed in an undulator magnetic field with amplitude $B_{\perp} \leq 0.17 \mathrm{~T}$. The electrodynamic system of the LF-section is composed of the two-mirror cavity consisting of 2D and 1D Bragg reflectors connected by a regular waveguide, and the wave deflector to deliver mm-wave to the HF-section. Both $1 \mathrm{D}$ and $2 \mathrm{D}$ reflectors are formed by two parallel conducting plates with corrugation of their surface in the form of parallel rectangular grooves and "chessboard" corrugation respectively. The $2 \mathrm{D}$-reflector of the cavity has the length $l_{2 D}=20 \mathrm{~cm}$ with its corrugation depth $a_{2 D}=220 \mu \mathrm{m}$ and the period of corrugation $d_{2 D}=0.4$ $\mathrm{cm}$. The main Fourier harmonic of the corrugation has the amplitude:

$$
A_{1,1}=16 a_{2 D}\left[\cos \left(h_{2 D} z-h_{2 D} x\right)+\right.
$$
$\left.+\cos \left(h_{2 D} z+h_{2 D} x\right)\right] / \pi^{2}$, where $h_{2 D}=2 \pi / d_{2 D}-x$ and $z$ components of translation vector. The same parameters for 1D-reflector are: the length $l_{1 D}=20 \mathrm{~cm}$, depth of corrugation $a_{1 D}=70 \mu$ mand its period $d_{1 D}=0.2 \mathrm{~cm}$. The amplitude of main Fourier harmonic is: $A_{1}=4 a_{1 D} \cos \left(h_{1 D} z\right) / \pi$, where $h_{1 D}=2 \pi / d_{1 D}$ - translation vector of grating.

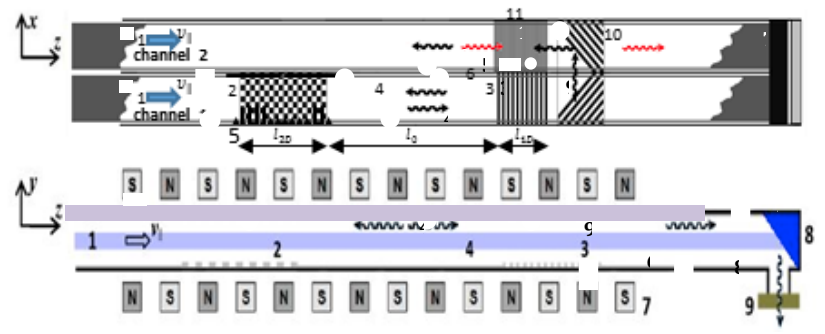

Fig. 1. Layout of the ELMI experiment to study the two-stage scheme for THz generation. 1 - parallel sheet electron beams, 2 -2D-Bragg reflector, 3 - 1D-reflector, 4 - regular waveguide section, 5 - baffle between channels, 6 - planar undulator (shown in the $y$-z plane), 7 - beam collector, 8 - output window, 9 - wave deflector, 10 - Bragg transformer of travelling to cutoff wave

\section{Wave deflector}

The main task for the design of the wave deflector is the achieving the high efficiencyof the radiation transmission from the LF-section to the HF-section and the required wave structure at the exit of the deflector.

To find the optimal configuration of the deflector, two main features of its corrugation were varied in the modeling: the shape of the corrugated region and the corrugation depth. In the result of the numerical solution of the coupled wave equations the optimal expression for the corrugation border was found: $z=2.69 \cdot \exp (|x| / 2.9)-3.58$ $\mathrm{cm}, x=-10 \div 10 \mathrm{~cm}, z=0 \div 13 \mathrm{~cm}$. Then the optimal depth of the corrugations was determinedin 3D full electro-dynamic calculations. Fig. 2 shows the results of modeling and measurements for the transformation of $\mathrm{H}_{10}$ mode passing in LF-section to the modes of HF-section for the corrugation depth of the deflector $370 \mu \mathrm{m}$.

Both 3D calculations and "cold" tests demonstrate the high transmission efficiency $(\sim 80 \%)$ from the LFsection to the HF-section in the band $74 \div 76 \mathrm{GHz}$. As to the mode purity, some parasitic conversion to highest modes increasing with the increase of the corrugation depth was observed. 


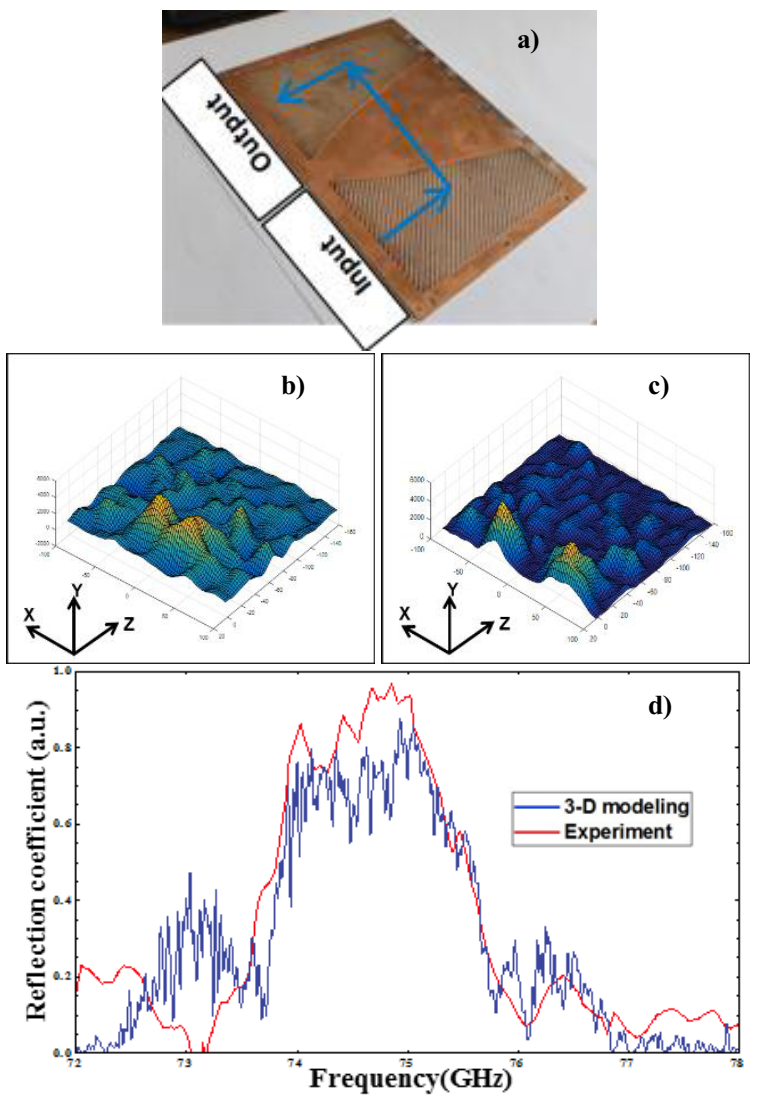

Fig. 2. (a) - photo of the wave deflector plate, (b),(c) - flux density distributions $P_{x}$ and $P_{z}$ in the plane (x,z),(d) - transmission efficiency of the deflector from input to outputmeasured in "cold" tests

\section{Wave Transformer}

In order to intensify the interaction of the beam with the mm-wave, the transformer of the travelling wave to the cutoff one is planned to be applied in HF-section. Such wave conversion will serve to some decreasing the frequency of scattered radiation to the range $0.6 \div 1 \mathrm{THz}$ that is more suitable for further mode selection by means of the cavity with advanced Bragg structures [5]. For this purpose, we developed 1D Bragg structure with one leap phase equal to $\pi$ in the center of the structure. Such transformer with sizes of its plates $l^{t r}=19.6 \mathrm{~cm}, l_{\perp}^{t r}=10 \mathrm{~cm}$ and the gap between them $a_{0}=0.6 \mathrm{~cm}$, has rectangular corrugation with the depth $a_{t r}=70 \mu \mathrm{m}$ and the period $d_{t r}=0.4 \mathrm{~cm}$ (see Fig. 3, a).According to 3D-modeling this wave structureprovides substantial increase of the scattering wave amplitude( $\sim 10$ times) but only in the narrow frequency band $75.05 \div 75.12 \mathrm{GHz}$ (see Fig. 3, b). This will make difficultiesfor ingress of the operating mode of LF-section in this band. To overcome them, new Bragg transformer with two leaps of phase is under development now that will allowobtaining more wider band with effective conversion to cutoff wave and larger gain factor.These conditions should provide more effective beamwave interaction resulting in the increase of the THzradiation power in the future experiments at the ELMI device.
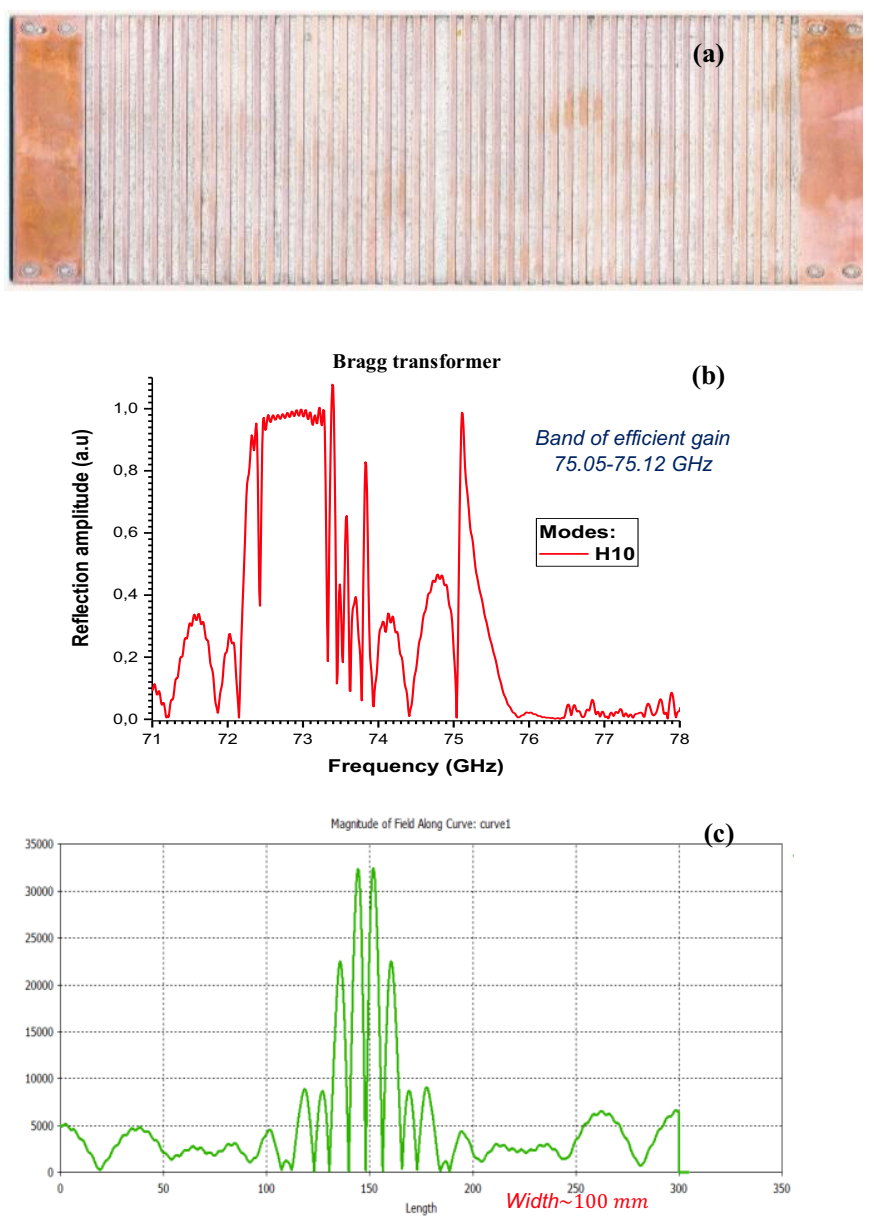

Fig. 3. (a) - photo of the Bragg transformer with aleap phase, (b) - reflection coefficient of Bragg transformer, obtained in 3D modeling, (c) - amplitude amplification

\section{Acknowledgements}

The work was partially supported by RSCF Project \#14-12-00610 and by the Ministry of Education and Science of the Russian Federation under the State Assignment Contract \#3002.

\section{References}

1. A.V. Arzhannikov et al. Generation of Powerful Narrow Band $75 \mathrm{GHz}$ Radiation in a Free Electron Maser with Two Dimensional Distributed Feedback// Tech. Phys. Lett. 2013. V. 39, No. 9. P. 801-804.

2. A.V. Arzhannikov et al.Intercavity scattering scheme for two-stage generationof submillimeter radiation on the base of planar 2DBragg FEM// Proc. of 6 Int. Workshop "Strong microwaves in plasma",N.Novgorod, Russia, 2005. P.228.

3. A.V. Arzhannikov et al.A traveling wave ring resonator with Bragg deflectors in a two-stage terahertz free electron laser// Tech. Phys. Lett. 2014. V. 40, No. 9. P. 730-734.

4. A.V. Arzhannikov et al. Using Two-Dimensional Distributed Feedback for Synhcronization of Radiation from two Parallel Sheet Electron Beams in Free Electron Maser// Phys. Rev. Lett. 2016. V.117, 114801.

5. A.V. Arzhannikov et al. Short-wavelength tunable Bragg reflectors based oncoupling of propagating and cutoff waves: Modeling and experimental studies//Appl. Phys. Lett. 2012. V. 101, 083507. 